\title{
Pulmonary gangrene and the air crescent sign
}

\author{
Jerome M Reich
}

\begin{abstract}
Background A study was carried out to increase familiarity with the aetiology, pathogenesis, and radiographic features that characterise pulmonary gangrene. Patients Four patients with one of the disorders vasoinvasive aspergillosis, infarcted tuberculous cavity, chronic necrotising aspergillosis, and gangrene due to Pseudomonas aeruginosa were selected because they showed the variations of the typical radiographic pattern and illustrated the pathogenesis. A fifth case is also presented, in which pulmonary gangrene was simulated by the invagination of a loculated pleural effusion into the wall of a contiguous lung abscess. Conclusions Evolution of a crescent or rim of air within a homogeneous shadow is the feature that both heralds the development and facilitates the recognition of pulmonary gangrene. It is most often the result of vascular thrombosis induced by the infecting organism. The outcome of treatment is often unfavourable, principally because of the severity of the predisposing systemic or local underlying disorder, although a delay in diagnosis, possibly due to unfamiliarity with the radiographic pattern, may have contributed to the adverse outcome in some instances.
\end{abstract}

(Thorax 1993;48:70-74)

Pulmonary gangrene comprises a subset of the radiographic pattern of a mass within a cavity in which both features are the consequence of tissue necrosis induced by infection. The distinctive pathogenesis, prognosis, and therapeutic implications set it apart from other causes of this pattern. The term "sphacelus," derived from the Greek sphakelos" (gangrene), was first used in 1575 by Banister. ${ }^{2}$ It was employed by Laennec ${ }^{3}$ to denote a necrotic mass within the cavity wall and more recently by Khan et al, who defined it as "a mass of sloughing, gangrenous, or necrotic matter."

The purpose of this communication is to illustrate the distinguishing clinical and radiographic features of pulmonary gangrene, and to attempt to provide an explanation for its development in each instance.

\section{Case histories}

CASE 1: ASPERGILLOMA COMPLICATING INVASIVE ASPERGILLOSIS

A 68 year old woman with erythroleukaemia developed widespread pulmonary infiltrates during a one week period of profound neutropenia (absolute neutrophil count $<100 / \mathrm{mm}^{3}$ ). The diffuse infiltrate resolved as the neutropenia responded to chemotherapy for her leukaemia, leaving a homogeneous opacity in the right upper lobe, which subsequently cavitated, revealing a mass surmounted by an air crescent (fig 1). Fibreoptic bronchoscopy and transbronchial biopsy were unhelpful. No diagnosis was made; the lesion continued to expand and the patient subsequently died. At necropsy a mass was identified that occupied much of the right upper lobe. It was composed of necrotic lung supplied by a large thrombosed vessel. The lung tissue, arterial wall, and thrombus were infiltrated by fungal hyphae. Similar elements, later identified as Aspergillus fumigatus, were found in the left upper lobe, liver, and spleen.

The rapid onset of diffuse pulmonary infiltration, the absence of the organism in the bronchial washings, and the necropsy evidence of extrapulmonary spread suggest that the initiating event was haematogenous dissemination. The resolution of the diffuse infiltrate coinciding with the appearance of the pulmonary gangrene can most easily be accounted for by the recovery of the neutrophil count, enabling the eradication of aspergilli in areas in which the blood supply remained intact. Release of neutrophilic proteolytic enzymes resulted in cavitation in the right upper lobe, where infarction due to vasoinvasive aspergillosis had already taken place. ${ }^{56}$

Pulmonary gangrene and the air crescent sign that accompanies it is most often caused by

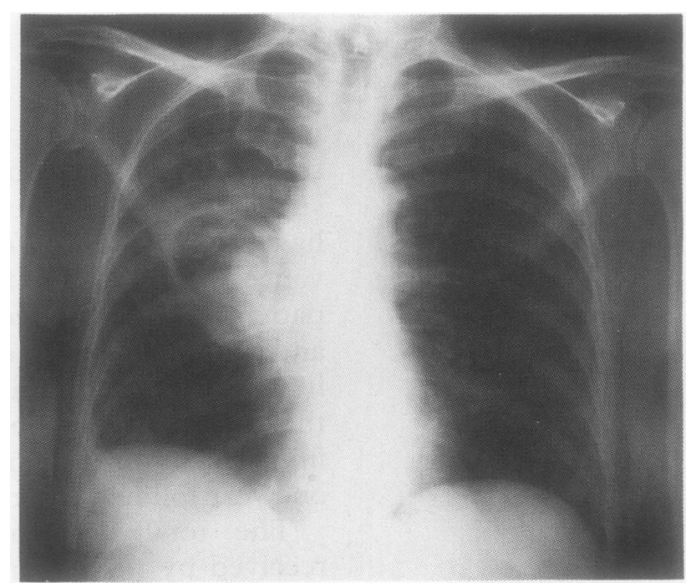

Figure 1 Case 1: The dense shadow to the right of the hilum, which was previously homogeneous, has undergone cavitation, revealing a mass surmounted by an air crescent. 

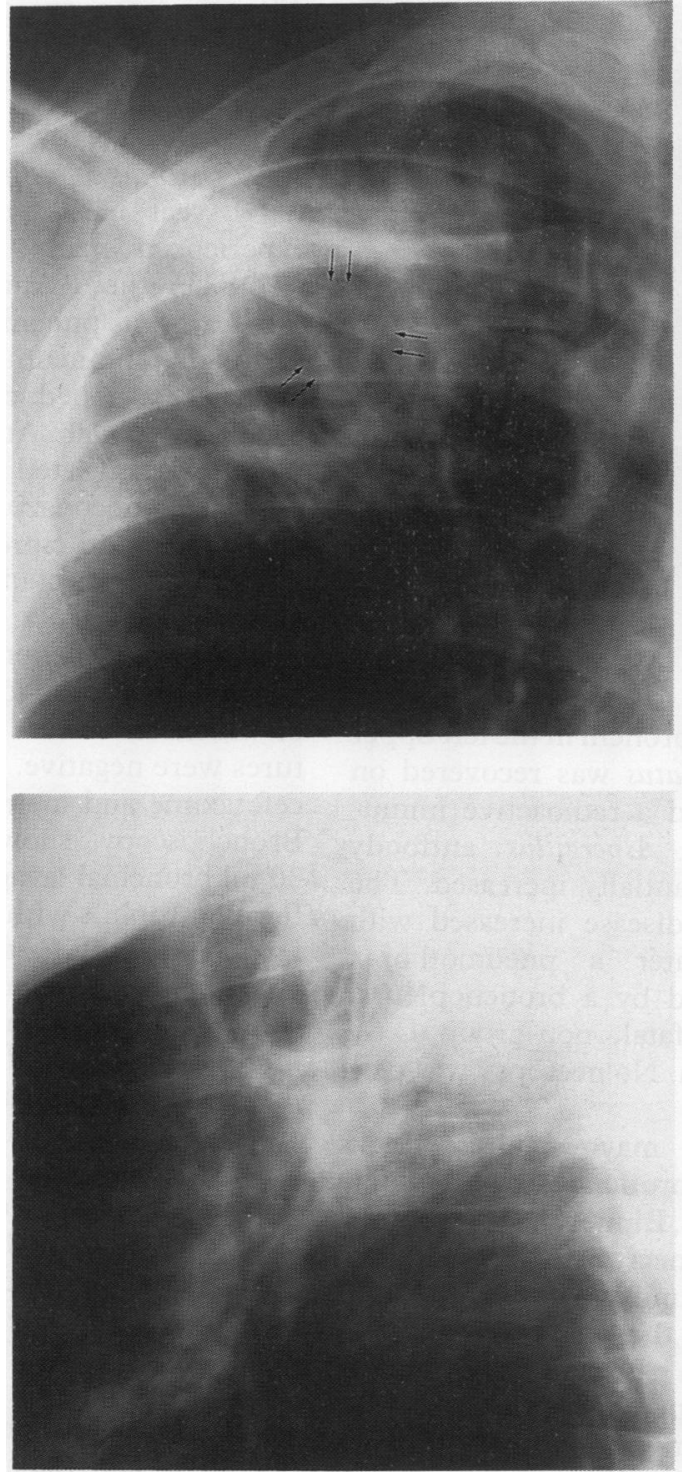

Figure 2 Case 2: (Top) View of $5 \mathrm{~cm}$ cavity of the right upper lobe with a small amount of pericavitary infiltration and a convoluted "cavity within a cavity" (arrows) thought to represent a slough of the interior aspect of the outer cavity. (Bottom) Lateral view of the cavity showing the concentric relation of the cavity and its slough.

angioinvasive pulmonary aspergillosis. ${ }^{78}$ Failure to recognise the diagnostic and therapeutic importance of this distinctive radiographic pattern may have dire consequences.

CASE 2: INFARCTED TUBERCULOUS CAVITY

A 39 year old black male patient, known to be tuberculin positive since childhood, developed fever and a cough productive of copious sputum two months before admission. Three years previously he underwent partial pancreatectomy to reduce pain caused by alcohol induced pancreatitis. Poorly controlled insulin dependent diabetes mellitus ensued. A chest radiograph (fig 2 ) showed an inner cavity wall surrounded successively by a rim of air, an outer concentric cavity wall, and pericavitary infiltration. Three sputum smears were negative for acid fast bacilli. Bronchoscopy showed erythema and small quantities of pus in a narrowed right upper lobe bronchus. Trans- bronchial biopsy of the inner cavity wall showed fragments of necrotic tissue that contained numerous acid fast bacilli. Bronchial washing revealed few acid fast bacilli and was culture positive for Mycobacterium tuberculosis. The patient responded favourably to treatment for tuberculosis.

A clinical diagnosis of tuberculosis in this patient with multiple risk factors, a strongly suggestive history, and a compatible chest radiograph was nearly discarded because of the repeated absence of stainable acid fast bacilli in sputum specimens. Two features support the interpretation that infarction of the tuberculous cavity, a rarely reported event, ${ }^{4}$ is likely to have taken place-namely, the presence of concentric cavitary walls that suggest a tissue slough and the necrotic tissue recovered from the inner cavity wall at transbronchial biopsy. Its occurrence may have reduced the number of organisms present in the sputum. Smear negative cavitary tuberculosis has not previously been associated with or attributed to cavitary infarction. Among the four patients with tuberculous pulmonary gangrene reported by Khan et al, ${ }^{4}$ three had a postmortem examination and were found to have extensive tuberculous pulmonary arteritis with secondary thrombosis. By contrast with case 2, all four had strongly positive sputum smears. All had very advanced disease and it is possible that the organisms identified on the smears may have arisen from regions of the lung unaffected by the infarction.

CASE 3: CHRONIC NECROTISING PULMONARY ASPERGILLOSIS COMPLICATING RADIATION INDUCED FIBROSIS

A 66 year old diabetic woman was admitted to hospital in 1986 for treatment of a bronchopleural fistula complicated by empyema. In 1978, she underwent radiotherapy of a large but localised breast cancer. Profound radiation fibrosis of both the breast and the underlying lung ensued. In 1982, pulmonary gangrene developed in the affected area (fig 3); and tomography showed very dilated airways. Fibreoptic bronchoscopy, undertaken to evaluate haemoptysis, showed pale, constric-

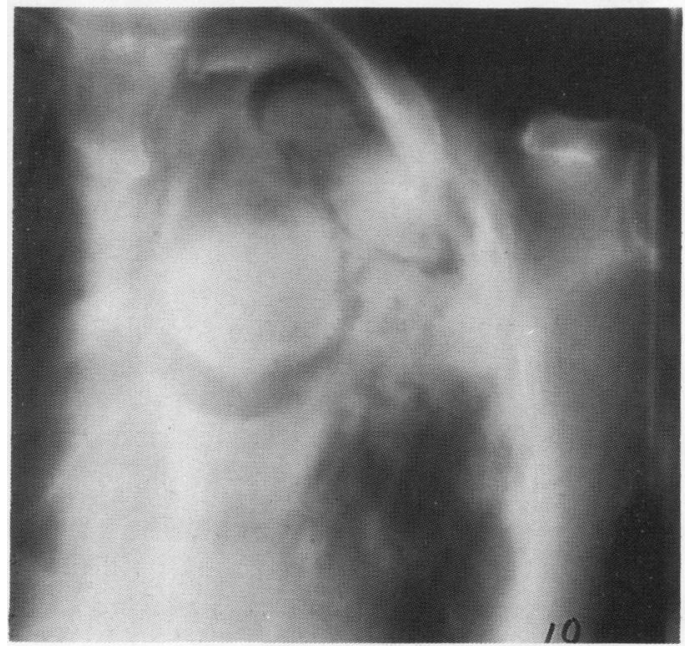

Figure 3 Case 3: Linear tomography of the left upper lobe showing a mass within a cavity. 
Figure 4 Case 4: Note the narrow rim of air surrounding the mass and demarcating it from the cavity wall.

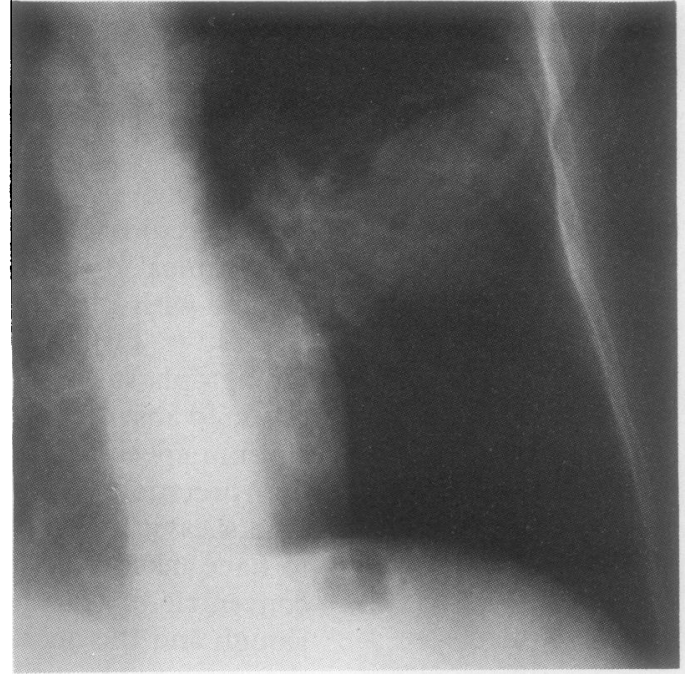

ted, and foreshortened bronchi in the left upper lobe. Aspergillus fumigatus was recovered on bronchial washing, and a radioactive immunoassay for specific Aspergillus antibody showed it to be substantially increased. The extent of pulmonary disease increased with time. Four years later a pneumothorax developed, complicated by a bronchopleural fistula that led to a fatal, non-group $D, \alpha$ streptococcal empyema. No necropsy was performed.

Aspergillus species may manifest their invasive potential in fibrotic areas of the lung, most often in the apices. Binder et al ${ }^{9}$ suggested the term "chronic necrotising pulmonary aspergillosis" for this unusual entity, which is intermediate between the angioinvasive pattern seen principally in patients with profound and protracted neutropenia and the relatively benign, non-invasive mycetoma that develops in patients with pre-existing cavities. Gefter et $a l^{10}$ described a similar appearance in patients who developed radiation induced pulmonary fibrosis. A possible explanation for this localised occurrence (it is not associated with diffuse pulmonary fibrosis) is that profound

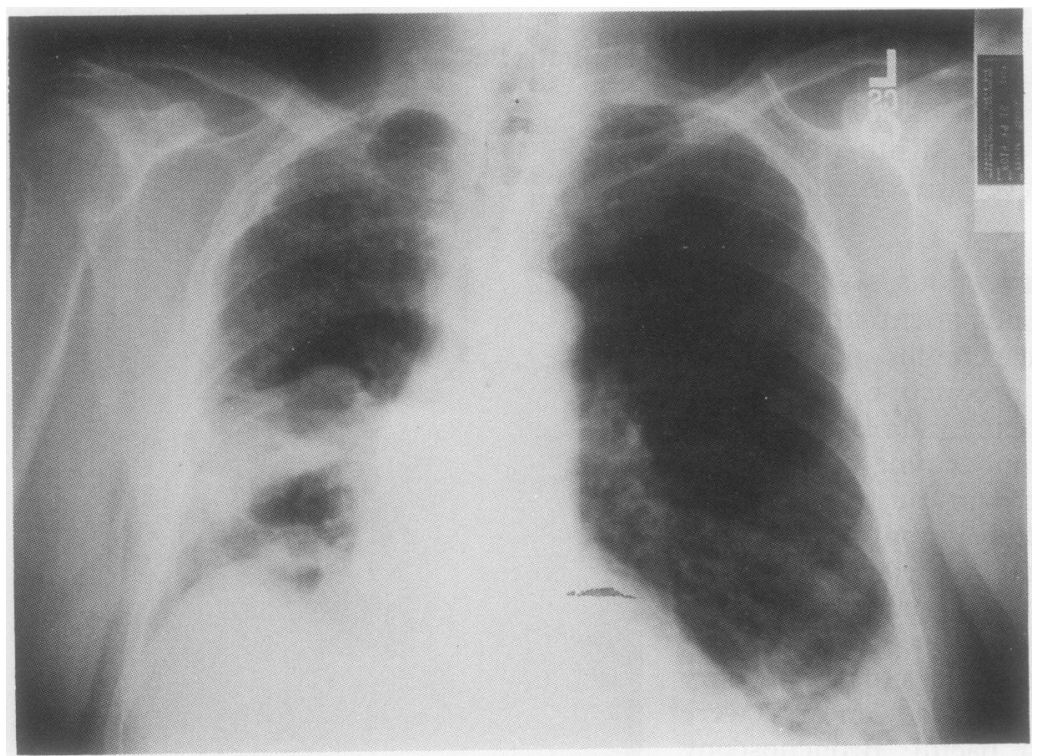

Figure 5 Case 5: A mass of eccentric location and irregular shape lies close to the inner wall of the cavity. A left lower lobe infiltrate is present. dilation of airways due to traction from the intense local scarring ${ }^{11}$ favours colonisation by Aspergillus fumigatus.

\section{CASE 4: PULMONARY GANGRENE DUE TO} PSEUDOMONAS AERUGINOSA IN AIDS

A 51 year old homosexual man, who had experienced many infections attributed to AIDS for three years, among them Pneumocystis carinii pneumonia, was admitted for treatment of pneumonia. Three days earlier he had developed chills, fever, and a nonproductive cough. At the time of admission his treatment consisted of dapsone, azidothymidine, and fluconazole, to which ethambutol, clofazimine and ciprofloxacin had been added because of suspected systemic Mycobacterium avium-intracellulare disease. His chest radiograph showed a homogeneous lingular infiltrate. His white blood cell count was $2.6 \times 10^{9} / 1$, of which $85 \%$ were granulocytes. Blood cultures were negative. Empirical treatment with cefotaxime and metronidazole was unhelpful. Bronchoscopy showed no abnormality. A $30 \mathrm{ml}$ bronchial lavage of the lingula showed a few neutrophils with no stainable organisms; a few colonies of Pseudomonas aeruginosa were recovered. Treatment was changed to piperacillin and tobramycin, but the infiltrate increased in size and pulmonary gangrene was indicated by the presence of a rim of air (fig 4). At thoracotomy, an $8 \times 6 \mathrm{~cm}$ intracavitary necrotic mass was found and a lobectomy performed. Microscopical examination of the surgical specimen showed mononuclear infiltration and extensive necrosis with no thrombi or vasculitic changes. Silver methenamine, Ziehl-Nielson, and Gram stains were all negative; tissue culture was positive only for Pseudomonas and the patient died with a postoperative empyema from which the same organism was recovered.

Pseudomonas species do not figure prominently among the pulmonary bacterial pathogens afflicting patients with AIDS. ${ }^{12}$ It seems likely that its role as a pulmonary pathogen was conditioned by the niche created by the antibiotic treatment received at the time the infection evolved. The suppressive effect of this regimen would also account for the absence of abnormalities at bronchoscopy and the few bacteria recovered in the bronchial wash specimen.

\section{CASE 5: PSEUDOSPHACELUS}

An 82 year old male cigarette smoker ( 40 packyears) was admitted to hospital in May 1990 for treatment of an unresolved right lower lobe pneumonia. He had lifelong asthma with occasional exacerbations requiring hospital care, but he was not dependent on oral steroid treatment. He took cimetidine daily for a peptic ulcer. Bronchoscopy showed mucoid secretions that grew normal flora. An organising pneumonia was suspected, antibiotics were stopped, and he was asked to return for a follow up chest radiograph. This showed that the area of infiltration had undergone cavitation. Lung abscess due to aspirátion of oral organisms was suspected, and he was treated with clindamycin. Sputum production became less and 
Figure 6 Case 5: Computed tomography lung window: the

"intracavitary mass" is an interlobar effusion that invaginated the

inferolateral aspect of the cavity wall.

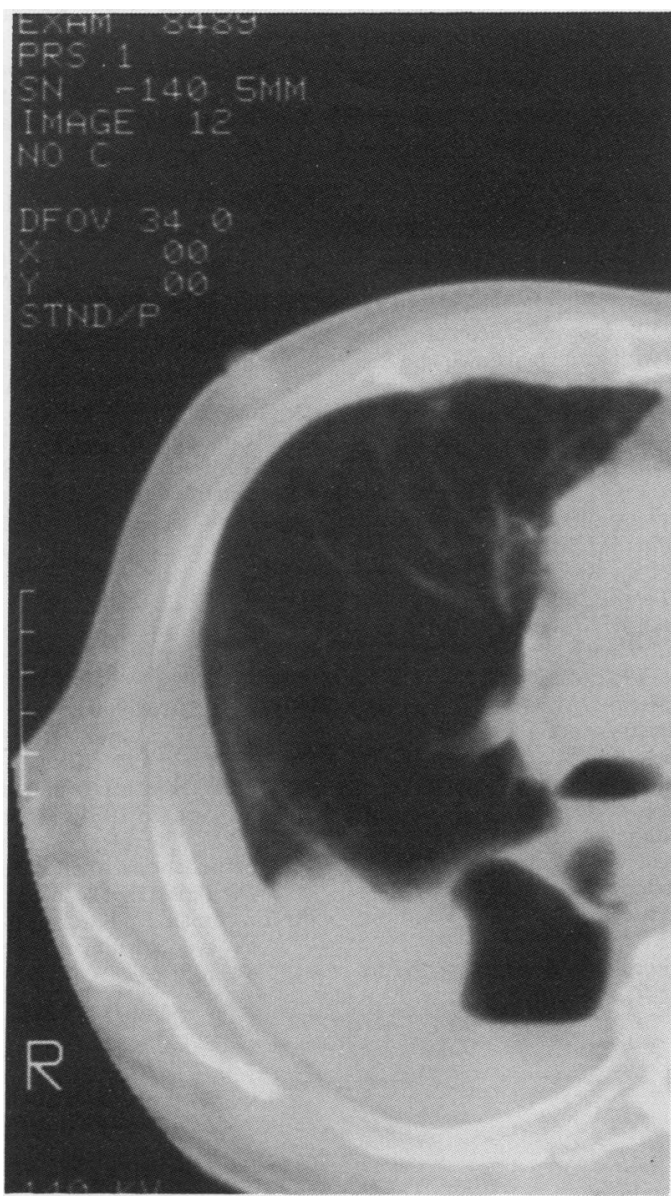

analogous to an osteomyelitic sequestrum. In this entity both cavity and mass are the consequence of infection induced tissue necrosis. Nearly synonymous terms are slough, ${ }^{13}$ massive sequestration of the lung,${ }^{14}$ gangrene with spontaneous amputation, ${ }^{15}$ spontaneous lobectomy, ${ }^{16} 17$ infarction of a pulmonary lobe, ${ }^{18}$ massive necrosis of lung, ${ }^{19}$ and massive pulmonary gangrene..$^{20-23}$

Pulmonary gangrene is most often encountered in invasive aspergillosis ${ }^{7-10}$ and with suppurative pneumonias due to Friedlander's bacillus, polymicrobial anaerobic organisms, ${ }^{1320}$ Pneumococcus, probably in association with other organisms, ${ }^{20}$ and Haemophilus influenza. ${ }^{4}$ Mucormycosis ${ }^{24}$ and tuberculosis ${ }^{4}$ are rarer causative agents. The common pathological feature in most cases is vascular thrombosis. The appearance of an air crescent was first recognised and applied to the description of aspergilloma by Pesle and Monod in $1954 .^{25}$

The common pathological feature shared by the first three cases is the loss or reduction of blood supply. In the angioinvasive form of aspergillosis, pulmonary infarction is the consequence of mycotic invasion of the vasovasora that results in infarction of the media and secondary thrombus formation. Tuberculous thrombosis of a pulmonary artery branch presumably accounted for the cavitary wall slough in case 2 . The chronic invasive form of aspergillosis seems to have a more complex mechanism: it seems likely that, owing to radiation induced impairment of blood supply, the invasive potential of Aspergilli colonising

the cavity diminished in size. Subsequently, a mass appeared within the cavity and an extensive infiltrate developed at the base of the opposite lung (fig 5). This led to the suspicion that a sphacelus was intermittently obstructing the bronchus draining the cavity, causing periodic spillage. Repeat bronchoscopy showed no new abnormalities. Lobectomy was considered but the patient responded to a change of antibiotic regimen to amoxacillinclavulanate. Computed tomography (fig 6) showed that the appearance of an intracavitary mass had been simulated by invagination of the abscess cavity by an interlobar extension of the pleural reaction. The process resolved with continued treatment except for a residual parenchymal and interlobar pleural scar.

In this patient, misinterpretation of an extracavitary mass as intracavitary almost led to unnecessary surgical intervention in a high risk patient. The repeated episodes of pneumonia and the original lung abscess were most likely due to episodes of aspiration.

\section{Discussion}

The phrase "mass within a cavity" is both cumbersome and lacking in specificity. It encompasses several entities that include mycetoma, Rasmussen aneurysm, and haematoma, in which a mass comes to occupy a pre or coexisting cavity, and pulmonary gangrene, first described by Laennec in $1826,{ }^{3}$ in which necrotic lung tissue is sloughed in a manner

\section{the ectatic bronchi is enhanced.}

In contrast the presence of proteolytic exotoxins seems the most likely explanation for the nearly identical radiographic pattern in the patient with a Pseudomonas induced pulmonary gangrene, as no vascular involvement was seen in the lung specimen. Most strains of this organism produce two or three proteolytic enzymes believed to cause the mixed haemorrhagic and infarction pattern of ecthyma gangrenosum. ${ }^{26-28}$ Whereas it seems reasonable to suppose that the pathological sequence in the lingula of this patient was analogous to ecthyma gangrenosum, the absence of previous reports of pulmonary gangrene attributable to Pseudomonas pulmonary infections, as contrasted with Friedlander's bacillus and polymicrobial anaerobic infections, ${ }^{13-23}$ casts doubt on the adequacy of this explanation.

Pseudosphacelus may be regarded as a radiographic curiosity as no previous reports exist of an intracavitary mass simulated by the fortuitous juxtaposition of an interlobar effusion and a contiguous cavitary structure. Careful analysis of the radiographic sequence enables it to be distinguished from pulmonary gangrene: the mass made its appearance after the abscess was fully developed and it did not conform in shape to the cavity it occupied.

I express my appreciation to Kyle Fuchs, MD and Kendall Barker, MD, for their many helpful modifications of the manuscript. 
1 Sphacelus. Oxford English Dictionary. 2nd ed. Oxford: Clarendon Press, 1989;XVI:203.

2 Banister J. A treatise of chyrurgerie. London: Thomas Marshe, 1575.

3 Laennec RTH. Traite de l'auscultation mediate et des maladies des poumons et du coeur; Tome premier, 2 nd ed. Paris: JS Chaude, 1826

4 Khan FA, Rehman M, Marcus P, Azueta V. Pulmonary gangrene occurring as a complication of pulmonary tuberculosis. Chest 1980;77:76-80.

5 Albeda SM, Talbot GH, Gerson SL, Miller WT, Cassileth PA. Pulmonary cavitation and massive hemoptysis in invasive pulmonary aspergillosis; influence of bone marrow recovery in patients with acute leukemia. $A m R e v$ Respir Dis 1985;131:115-20.

6 Janoff A, Sandhaus RA, Hospelhorn VD, Rosenberg R. Digestion of lung proteins by human leukocyte granules in vitro. Proc Soc Exp Biol Med 1972;140:516-9.

7 Curtis AM, Walker Smith GJ, Ravin CE. Air crescent sign of invasive aspergillosis. Radiology 1979;133:17-21.

8 Slevin ML, Knowles GK, Phillips MJ, Stansfeld AG, Lister TA. The air crescent sign of invasive pulmonary aspergillosis in acute leukaemia. Thorax 1982;37:554-5.

9 Binder RE, Faling LJ, Pugnatch RD, Mahasaen C, Snider GL. Chronic necrotizing pulmonary aspergillosis: a dis-
crete clinical entity. Medicine 1982;61:109-24.

10 Gefter WB, Weingrad TR, Epstein DM, Ochs RH, Miller WT “"Semi-invasive" pulmonary aspergillosis. Radiology 1981;140:313-21

11 Westcott JL, Cole SR. Traction bronchiectasis in end-stage pulmonary fibrosis. Radiology 1986;161:665-9.

12 Murray J. State of the art: Pulmonary infectious complications of human immunodeficiency virus infection; part 1. Am Rev Respir Dis 1990;141:1356-72.

13 Brock RC. Lung abscess. Oxford: Blackwell Scientific Publications, 1952.

14 Brock RC. Studies in lung abscess. Guy's Hospital Report 1946;95:40-7.
15 Lenoble E, Jegat Y. Gangrene limitee a'une portion du poumon droit avec amputation spontanee du lobe superieur. Bulletins et memoires de la Societe Medicale des Hospitaux de Paris 1921;45:1534-8.

16 Humphreys DR. Spontaneous lobectomy. BMJ 1945;2: 185-6.

17 Taylor JW. Spontaneous lobectomy. BMJ 1954;2:500-1.

18 Rawson AJ, Cocke JA. Infarction of an entire pulmonary lobe with subsequent aseptic softening causing sterile hemopneumothorax. Am J Med Sci 1947;214:520-4.

19 Thomas JH, Brewster OM. Friedlander's pneumonia with massive necrosis of lung. $B M J 1952 ; 2: 817-8$.

20 Danner PK, McFarland DR, Felson B. Massive pulmonary gangrene. American Journal of Roentgenology, Radium Therapy, and Nuclear Medicine 1968;103:548-54.

21 Gutman E, Pongdee O, Park YS. Massive pulmonary gangrene. Radiology 1973;107:293-4.

22 Knight L, Fraser RG, Robson HG. Massive pulmonary gangrene: a severe complication of klebsiella pneumonia. Can Med Assoc J 1975;112:196-8.

23 Cameron DI, Stewart JD, Slack RC, Lewis CL. Massive pulmonary gangrene [letter to editor]. Can Med Assoc J 1975;112:1290.

24 Reich JM, Renzetti AD. Pulmonary phycomycosis; report of a case of bronchocutaneous fistula formation and pulmonary arterial mycothrombosis. Am Rev Respir Dis 1970;102:959-63.

25 Pesle GD, Monod O. Bronchiectasis due to aspergilloma. Dis Chest 1954;125:172-83.

26 Liu PV. Extracellular toxins of pseudomonas aeruginosa. $J$ Infect Dis 1974;130(suppl O):S94-9.

27 Liu PV. The roles of various fractions of pseudomonas in its pathogenesis. ii. Effects of lecithinase and protease. J Infect Dis 1966;116:112-6.

28 Hitschman F, Kreilich K Zur Pathogenese des Bacillus Pyocyaneus und zur Aetiologie des Ekthyma Gangraenosum. Wiener Klinische Wochenschrift 1897;10:1093-101. 